\title{
Operative outcomes of robotic partial nephrectomy
}

\section{A report of the first 101 cases from a single center in Saudi Arabia}

\author{
Raouf M. Seyam, MD, Mohammed M. Alalawi, MD, Waleed K. Alkhudair, FRCS(C), Hassan M. Alzahrani, MD, \\ Raed A. Azhar, FRCS(C), Khalid I. Alothman, FRCS(C), Turki O. Al-Hussain, MD, Mohammed F. Alotaibi, FRCS(C).
}

\begin{abstract}
الأهداف : اكتسب استئصال الكملية الجزئي بالروبوت شعبية في المبازي

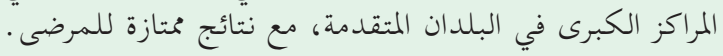

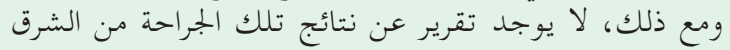

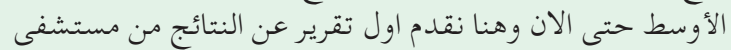

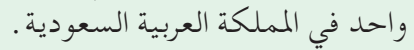

الطريقة: راجعنا بأثر رجعي الحالات المتتالية للمرضى الذين

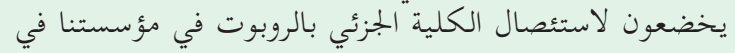

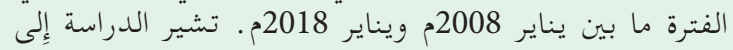

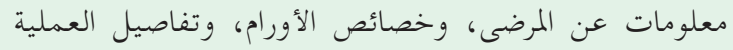

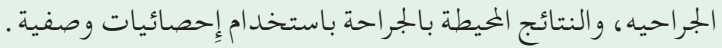

النتائج : خضع 101 مريض للجراحة خلال فترة الدراسة. كان

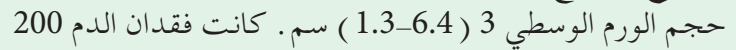

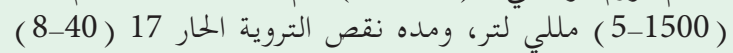

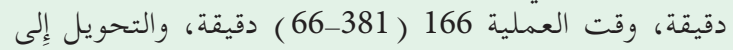

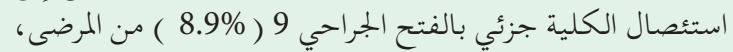

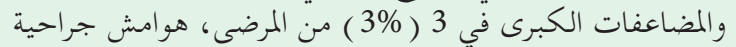

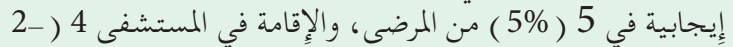

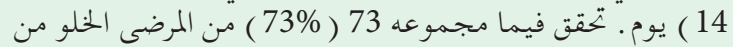

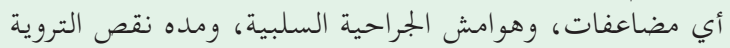

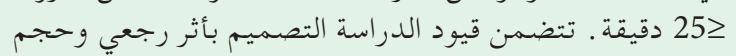

المجموعة الصغيرة.

الخاتمة: في هذه السلسلة، تشابهت التجربة الأولية لاستئصال

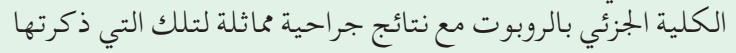

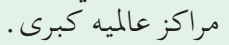

Objectives: To report robotic partial nephrectomy (RPN) outcomes from a single tertiary hospital in Saudi Arabia.

Methods: We retrospectively reviewed consecutive cases of patients undergoing RPN at King Faisal Specialist Hospital and Research Center, Riyadh,
Kingdom of Saudi Arabia, between January 2008 and January 2018. The study reports patient's demographics, tumor characteristics, operative details, and perioperative outcomes, using descriptive statistics of median and range values.

Results: One hundred and one patients underwent RPN during the study period. Average tumor size was $3(1.3-6.4) \mathrm{cm}$ and average radius exophytic nearness anterior/posterior location (RENAL) score was 6 (4-10). Perioperative parameters were blood loss $200(5-1500) \mathrm{ml}$ and warm ischemia time 17 (8-40) minutes, excluding off-clamp surgery in 12 (11.9\%); operative time was 166 (66-381) minutes. Conversion to open partial nephrectomy occurred in $9(8.9 \%)$ patients, major complications in $3(3 \%)$ patients, positive surgical margins in $5(5 \%)$ patients, and the hospital stay was 4 (2-14) days. A total of 73 (73\%) patients achieved a trifecta of freedom from any complication, negative surgical margins, and ischemia time $\leq 25$ minutes. Study limitations included the retrospective design and small cohort size.

Conclusions: The initial experience of robotic partial nephrectomy was associated with a surgical outcome comparable to that reported by higher-volume centers.

Saudi Med J 2019; Vol. 40 (1): 33-40 doi: 10.15537/smj.2019.1.22782

From the Department of Urology (Seyam, Alalawi, Alkhudair, Alzahrani, Alothman, Alotaibi), King Faisal Specialist Hospital and Research Center, from the Department of Pathology (Al-Hussain), King Faisal Specialist Hospital and Research Center, Riyadh, and from the Department of Urology (Azhar), King Abdulaziz University, Jeddah, Kingdom of Saudi Arabia.

Received 18th September 2018. Accepted 28th November 2018.

Address correspondence and reprint request to: Dr. Mohammed F. Alotaibi, Consultant, Department of Urology, King Faisal Specialist Hospital and Research Center, Riyadh, Kingdom of Saudi Arabia. E-mail: otaibim@kfshrc.edu.sa

ORCID ID: orcid.org/0000-0002-5498-5795 
$\mathrm{R}$ obotic-assisted partial nephrectomy (RPN) has exhibited superiority to laparoscopic partial nephrectomy (LPN) for small renal tumors; thus, the recent trend in minimally invasive partial nephrectomy has shifted toward RPN. ${ }^{1-3}$ Robotic-assisted partial nephrectomy embrace a brief learning curve and has advantages in all the parameters of warm ischemia time (WIT), conversion to open surgery, surgical margins, perioperative complications, change of renal function, and length of hospital stay (LOS). ${ }^{1-4}$ We report the perioperative outcomes of RPN surgery in a single center in Saudi Arabia.

Methods. This is a retrospective study of the electronic records of patients who underwent RPN at King Faisal Specialist Hospital and Research Center, Riyadh, Kingdom of Saudi Arabia, between January 2008 and January 2018. The Institution Review Board approved the project. The study was conducted according to principles of Helsinki Declaration.

Inclusion criteria involved all patients who underwent RPN of any age, gender or indication. No exclusion criteria were applied.

Surgical technique. The $\mathrm{Si}$ robotic system (da Vinci ${ }^{\oplus}$ Surgical System, da Vinci ${ }^{\oplus} \mathrm{Si}$, USA) was used for all RPNs. A 3-arm or 4-arm robotic approach was used according to the surgeon's preference. The kidney was mobilized entirely outside Gerota's fascia, and the tumor with intact peri-renal fat was localized and scored using electrocautery and the adjacent kidney de-fatted. Intraoperative laparoscopic ultrasound was used in some of the recent cases. Sharp resection of the tumor was performed using robotic scissors. Sutured renorrhaphy was carried out in 2 stages: the bed of the resection was sutured in a running fashion, using monofilament absorbable sutures or self-locking barbed suture (V-Loc 90; Covidien, Mansfield, Massachusetts) according to the surgeon's preference, and then the partial nephrectomy defect was closed by interrupted monofilament suture. All cases were video-recorded for quality assurance and review if necessary. The reported parameters included patients' demographics; tumor characteristics, including size, location, radius exophytic nearness anterior/posterior location (RENAL) nephrometry score, stage, histopathology type, grade, and surgical margin; operative details,

Disclosure. Authors have no conflict of interests, and the work was not supported or funded by any drug company. including operative time, WIT, estimated blood loss (EBL); and postoperative outcomes, including day one serum creatinine, estimated glomerular filtration rate (eGFR) change, LOS, and complications. We used the Modification of Diet in Renal Disease Study Group equation (MDRD) to calculate eGFR. ${ }^{5}$ To assess the progress of learning of the surgeons, we divided the patients into 2 nearly equal chronological groups and compared their characteristics and perioperative outcomes. To review the literature, we conducted a PubMed search for citations up to December 2017 using the term "robotic partial nephrectomy" and restricted the output to "English Language and Human". We compared perioperative outcomes of publications that included a number of patients similar to our series.

We used the program SPSS version 20 (IBM Corporation, USA) for the statistical analysis. We utilized descriptive statistics reporting the median, standard deviation (SD), minimum and maximum values for continuous variables and numbers and percentages for categorical values. In subgroup analysis, we compared continuous variables with analysis of variance reporting mean and SD values and for categorical values, we utilized Fisher exact test. Significant results were reported if $p<0.05$.

Results. A total of 101 consecutive patients underwent RPN (Table 1), between January 2008 and January 2018. Four urologists without prior experience in RPN performed 85 procedures (Figure 1). Patients were diagnosed with a small renal mass either incidentally $(\mathrm{n}=72 ; 71.3 \%)$ or due to symptoms (pain or hematuria; $\mathrm{n}=29 ; 28.7 \%)$. Clinical staging showed that $84(84 \%)$ patients had a stage T1aN0M0 tumor, whereas 16 patients had a stage of T1bN0M0. Eighty tumors $(79.2 \%)$ were solid and $20(19.8 \%)$ were complex renal cysts. One patient had RPN for a nonfunctioning upper renal moiety.

Surgical outcomes of RPN are shown in Table 1. Four patients (4\%) needed an intraoperative transfusion of a single unit of blood. Nine patients $(8.9 \%)$ were converted to open partial nephrectomy because of bleeding or lack of progress in dissection. During RPN, 12 patients (11.9\%) underwent no renal vascular clamping, whereas warm ischemia occurred in 89 patients $(88.1 \%)$ by selective arterial clamping. Preoperatively, 96 (95\%) patients had an eGFR value greater than $60 \mathrm{ml} / \mathrm{min} / 1.77 \mathrm{~m}^{2}$; postoperatively, none of these patients experienced a decrease in eGFR below $60 \mathrm{ml} / \mathrm{min} / 1.77 \mathrm{~m}^{2}$. Of all patients; however, 16 $(15.8 \%)$ experienced a decrease in eGFR of $\leq 15 \%$. 
Table 1 - Patient's characteristics and global surgical outcome $(\mathrm{N}=101)$.

\begin{tabular}{|c|c|c|c|c|}
\hline Characteristics & \multicolumn{4}{|c|}{ Number of patients (\%) } \\
\hline \multicolumn{5}{|l|}{ Gender } \\
\hline Female & \multicolumn{4}{|c|}{$42(41.6)$} \\
\hline Male & \multicolumn{4}{|c|}{$59(58.4)$} \\
\hline \multicolumn{5}{|l|}{ Renal score ${ }^{t}$} \\
\hline Renal score 4-6 & \multicolumn{4}{|c|}{$68(67.3)$} \\
\hline Renal score $\geq 7$ & \multicolumn{4}{|c|}{$32(31.7)$} \\
\hline \multicolumn{5}{|l|}{ Weight } \\
\hline Normal or overweight & \multicolumn{4}{|c|}{$52(51.5)$} \\
\hline Obese $(\mathrm{BMI}>30)$ & \multicolumn{4}{|c|}{$49(48.5)$} \\
\hline \multicolumn{5}{|l|}{ Histopathology } \\
\hline CRCC & \multicolumn{4}{|c|}{$57(56.4)$} \\
\hline AML & \multicolumn{4}{|c|}{$8 \quad(7.9)$} \\
\hline Oncocytoma & \multicolumn{4}{|c|}{$7 \quad(6.9)$} \\
\hline Papillary carcinoma & \multicolumn{4}{|c|}{$14(13.9)$} \\
\hline Chromophobe & \multicolumn{4}{|c|}{$10 \quad(9.9)$} \\
\hline Other benign & \multicolumn{4}{|c|}{$5(5.0)$} \\
\hline \multirow[t]{2}{*}{ Positive surgical margin } & \multicolumn{4}{|c|}{$5(5.0)$} \\
\hline & Median & Min & Max & SD \\
\hline Weight in (KG) & 81.6 & 37.5 & 143.8 & 19 \\
\hline BMI & 30 & 16.2 & 45.3 & 6 \\
\hline Age at diagnosis (years) & 47.2 & 21 & 77.3 & 13.7 \\
\hline $\begin{array}{l}\text { Size of lesion (Maximum } \\
\text { diameter in } \mathrm{cm} \text { ) }\end{array}$ & 3 & 1.3 & 6.4 & 1 \\
\hline Renal score ${ }^{*}$ & 6 & 4 & 10 & 1.7 \\
\hline Procedure time (minutes) & 166 & 66 & 381 & 57.4 \\
\hline $\mathrm{EBL}(\mathrm{ml})$ & 200 & 5 & 1500 & 213.4 \\
\hline WIT (minutes) $\dagger$ & 17 & 8 & 40 & 6.1 \\
\hline Postoperative duration (days) & 4.00 & 2 & 14 & 1.423 \\
\hline Preoperative creatinine $(\mu \mathrm{mol} / \mathrm{l})$ & 76 & 38 & 148 & 22.2 \\
\hline $\begin{array}{l}\text { 1st postoperative creatinine } \\
(\mu \mathrm{mol} / \mathrm{l})\end{array}$ & 81 & 42 & 178 & 24.5 \\
\hline Preoperative eGFR $\mathrm{ml} / \mathrm{m}^{2}$ & 93.5 & 45.0 & 180.5 & 24.3 \\
\hline Postoperative eGFR $\left(\mathrm{ml} / \mathrm{m}^{2}\right)$ & 88.7 & 34.8 & 166.4 & 23.7 \\
\hline Decrease in eGFR $\left(\mathrm{ml} / \mathrm{m}^{2}\right)$ & 4.3 & -38.6 & 47.1 & 15.4 \\
\hline Decrease in eGFR \% & 5.9 & -44.7 & 42.6 & 14.8 \\
\hline \multicolumn{5}{|c|}{$\begin{array}{l}\text { AML - angiomyolipoma, BMI - body mass index, CCRCC - clear cell } \\
\text { renal cell carcinoma, EBL - estimated blood loss, eGFR - estimated } \\
\text { glomerular filtration rate, WIT - warm ischemia time, *one patient had } \\
\text { RPN for non-functioning upper moiety and is not included. } \\
{ }^{\dagger} 12 \text { of } 89 \text { patients had no vascular clamping. }\end{array}$} \\
\hline
\end{tabular}

Table 2 shows a comparison between patients without and with a decrease in eGFR of $\leq 15 \%$. In patients who demonstrated the decrease, the only significant risk factors were a longer procedure time and the presence of a complication. This decrease occurred though there was a significantly better preoperative eGFR in those patients. Pathological examination of the tumors revealed $68 \mathrm{pT} 1 \mathrm{a}(68 \%), 8 \mathrm{pT} 1 \mathrm{~b}(8 \%), 6$ pT3a (6\%), and 19 benign lesions (18.8\%), including 8 angiomyolipomas (7.9\%), 7 oncocytomas (6.9\%), and 4 other lesions (4\%). A positive surgical margin was documented in 5 cases $(5 \%)$. One patient had a tumor rupture/spillage; this patient remained free of disease after 30 months.
Table 2 - Comparison between cases based on a postoperative decrease of eGFR $\geq 15 \%$.

\begin{tabular}{|c|c|c|c|c|c|}
\hline \multirow[t]{3}{*}{ Variables } & \multicolumn{5}{|c|}{ Decrease of eGFR $\geq 15 \%$} \\
\hline & \multicolumn{2}{|r|}{ No } & \multicolumn{2}{|c|}{ Yes } & \multirow{2}{*}{$P$-value } \\
\hline & & n $\quad(\%)$ & & $(\%)$ & \\
\hline Gender & & & & & 0.778 \\
\hline Female & & $35(35)$ & & $7 \quad(7)$ & \\
\hline Male & & $51(50)$ & $\varepsilon$ & $8 \quad(8)$ & \\
\hline Conversion to open & & & & & 0.129 \\
\hline No & & $80(79)$ & & $2(12)$ & \\
\hline Yes & & $6(6)$ & & $3(3)$ & \\
\hline Transfusion & & & & & 0.104 \\
\hline No & & $84(83)$ & & $3(13)$ & \\
\hline Yes & & $2(2)$ & & $2(2)$ & \\
\hline Off clamp technique & & & & & 0.380 \\
\hline No & & $77(76)$ & & $2(12)$ & \\
\hline Yes & & $9 \quad(9)$ & & $3(3)$ & \\
\hline Surgical margins & & & & & 1.000 \\
\hline Negative & & $81(81)$ & & $4(14)$ & \\
\hline Positive & & $5(5)$ & c & $(0)$ & \\
\hline Minor complication & & & & & 0.013 \\
\hline No & & $76(75)$ & s & (9) & \\
\hline Yes & & $10(10)$ & c & $6(6)$ & \\
\hline Major complication & & & & & 1.000 \\
\hline No & & $83(82)$ & & $5(15)$ & \\
\hline Yes & & $3(3)$ & $c$ & $(0)$ & \\
\hline \multicolumn{6}{|l|}{ Any complication } \\
\hline No & & $74(73)$ & s & (9) & 0.025 \\
\hline Yes & & $12(12)$ & c & $6(6)$ & \\
\hline Trifecta achieved ${ }^{*}$ & & & & & 0.194 \\
\hline No & & $65(65)$ & $\varepsilon$ & $8(8)$ & \\
\hline Yes & & $21(21)$ & c & $6(6)$ & \\
\hline \multicolumn{6}{|l|}{ Histopathology } \\
\hline Benign & & $19(19)$ & 2 & $2(2)$ & 0.731 \\
\hline \multirow[t]{2}{*}{ Malignant } & & $67(66)$ & & $3(13)$ & \\
\hline & $\mathrm{n}$ & Mean \pm SD & $\mathrm{n}$ & Mean \pm SD & \\
\hline BMI & 86 & $30.5 \pm 5.9$ & 15 & $28.3 \pm 6.1$ & 0.192 \\
\hline $\begin{array}{l}\text { Age at diagnosis } \\
\text { (years) }\end{array}$ & 86 & $49.1 \pm 13.7$ & 15 & $46.5 \pm 13.8$ & 0.507 \\
\hline $\begin{array}{l}\text { Size of lesion }(\max \\
\text { diameter in } \mathrm{cm})\end{array}$ & 86 & $3.0 \pm 1.1$ & 15 & $3.3 \pm 1.0$ & 0.303 \\
\hline Renal score $^{\dagger}$ & 86 & $5.7 \pm 1.7$ & 14 & $6.5 \pm 1.6$ & 0.102 \\
\hline $\begin{array}{l}\text { Procedure time } \\
\text { (minutes) }\end{array}$ & 86 & $161.2 \pm 42.6$ & 152 & $219.7 \pm 90.8$ & 0.000 \\
\hline $\mathrm{EBL}(\mathrm{ml})$ & 86 & $253.8 \pm 215.5$ & 152 & $271.7 \pm 207.2$ & 0.767 \\
\hline WIT (minutes) & 77 & $17.4 \pm 5.6$ & 12 & $18.7 \pm 8.8$ & 0.512 \\
\hline $\begin{array}{l}\text { Preoperative eGFR } \\
\mathrm{ml} / \mathrm{m}^{2}\end{array}$ & 86 & $93.9 \pm 22.3$ & 151 & $09.1 \pm 31.1$ & 0.025 \\
\hline $\begin{array}{l}\text { Postoperative eGFR } \\
\left(\mathrm{ml} / \mathrm{m}^{2}\right)\end{array}$ & 86 & $92.7 \pm 23.3$ & 15 & $80.9 \pm 24.2$ & 0.075 \\
\hline $\begin{array}{l}\text { Decrease in eGFR } \\
\left(\mathrm{ml} / \mathrm{m}^{2}\right)\end{array}$ & 86 & $1.2 \pm 11.9$ & 15 & $28.1 \pm 13.0$ & 0.000 \\
\hline Decrease in eGFR \% & 86 & $0.8 \pm 12.4$ & 15 & $25.4 \pm 8.9$ & 0.000 \\
\hline \multicolumn{6}{|c|}{$\begin{array}{l}\text { BMI - body mass index, EBL - estimated blood loss, eGFR - estimated } \\
\text { glomerular filtration rate, WIT - warm ischemia time, *Trifecta - freedom } \\
\text { of any complication, negative surgical margin and ischemia time } \leq 25 \text { min. } \\
{ }^{\dagger} \text { one patient had robotic partial nephrectomy (RPN) for non-functioning } \\
\text { upper moiety and is not included. }{ }^{\ddagger} 12 \text { of } 89 \text { patients had no vascular } \\
\text { clamping }\end{array}$} \\
\hline
\end{tabular}


Table 3 shows a comparison between patients without and with a PSM. The only significant difference was a higher mean age in the PSM group. Postoperatively, minor complications (Clavien-Dindo grade I-II) were encountered in $16(15.8 \%)$ patients. Only 3

Table 3 - A comparison between cases with positive and negative surgical margins.

\begin{tabular}{|c|c|c|c|c|c|}
\hline \multirow[t]{2}{*}{ Variables } & \multicolumn{5}{|c|}{ Surgical margins } \\
\hline & \multicolumn{2}{|r|}{$\begin{array}{l}\text { Negative } \\
\text { n (\%) }\end{array}$} & \multicolumn{2}{|r|}{$\begin{array}{l}\text { Positive } \\
\text { n (\%) }\end{array}$} & $P$-value \\
\hline Gender & & & & & 0.646 \\
\hline Female & & $40(40)$ & & $1(1)$ & \\
\hline Male & & $55(55)$ & & $4(4)$ & 1.000 \\
\hline \multicolumn{6}{|l|}{ Conversion to open } \\
\hline No & & $86(86)$ & & $5(5)$ & 1.000 \\
\hline Yes & & $9(9)$ & & $0(0)$ & \\
\hline Transfusion & & & & & 0.449 \\
\hline No & & $91(91)$ & & $5(5)$ & \\
\hline Yes & & $4(4)$ & & $0(0)$ & \\
\hline \multicolumn{6}{|l|}{$\begin{array}{l}\text { Off clamp } \\
\text { technique }\end{array}$} \\
\hline No & & $85(85)$ & & $4(4)$ & \\
\hline Yes & & $10(10)$ & & $1(1)$ & \\
\hline \multicolumn{6}{|l|}{$\begin{array}{l}\text { Minor } \\
\text { complication }\end{array}$} \\
\hline No & & $80(80)$ & & $4(4)$ & 1.000 \\
\hline Yes & & $15(15)$ & & $1(1)$ & \\
\hline \multicolumn{6}{|l|}{ Major complication } \\
\hline No & & $92(92)$ & & $5(5)$ & 1.000 \\
\hline Yes & & $3(3)$ & & $0(0)$ & \\
\hline \multicolumn{6}{|l|}{ Any complication } \\
\hline No & & $78(78)$ & & $4(4)$ & 1.000 \\
\hline Yes & & $17(17)$ & & $1(1)$ & \\
\hline \multicolumn{6}{|l|}{ Histopathology } \\
\hline Benign & & $20(20)$ & & $0(0)$ & 0.580 \\
\hline Malignant & & $75(75)$ & & $5(5)$ & \\
\hline Variables & $\mathrm{n}$ & Mean \pm SD & $\mathrm{n}$ & Mean \pm SD & \\
\hline BMI & 95 & $30.3 \pm 5.9$ & 5 & $30.7 \pm 5.1$ & 0.900 \\
\hline $\begin{array}{l}\text { Age at diagnosis } \\
\text { (years) }\end{array}$ & 95 & $48.2 \pm 13.5$ & 5 & $61.2 \pm 10.4$ & 0.038 \\
\hline $\begin{array}{l}\text { Size of lesion (max } \\
\text { diameter in } \mathrm{cm})\end{array}$ & 95 & $3.0 \pm 1.1$ & 5 & $3.6 \pm 0.5$ & 0.250 \\
\hline Renal score $^{*}$ & 95 & $5.8 \pm 1.7$ & 5 & $5.4 \pm 1.3$ & 0.568 \\
\hline $\begin{array}{l}\text { Procedure time } \\
\text { (minutes) }\end{array}$ & 95 & $169.2 \pm 54.7$ & 5 & $151.0 \pm 30.4$ & 0.464 \\
\hline $\mathrm{EBL}(\mathrm{ml})$ & 95 & $257.9 \pm 215.1$ & 5 & $250.0 \pm 217.9$ & 0.936 \\
\hline WIT (minutes) ${ }^{\dagger}$ & 85 & $17.6 \pm 6.2$ & 4 & $17.0 \pm 4.2$ & 0.846 \\
\hline $\begin{array}{l}\text { Preoperative eGFR } \\
\mathrm{ml} / \mathrm{m}^{2}\end{array}$ & 95 & $96.6 \pm 23.9$ & 5 & $76.2 \pm 9.4$ & 0.061 \\
\hline $\begin{array}{l}\text { Postoperative } \\
\text { eGFR }\left(\mathrm{ml} / \mathrm{m}^{2}\right)\end{array}$ & 95 & $91.8 \pm 24.0$ & 5 & $72.2 \pm 5.4$ & 0.071 \\
\hline $\begin{array}{l}\text { Decrease in eGFR } \\
\left(\mathrm{ml} / \mathrm{m}^{2}\right)\end{array}$ & 95 & $4.8 \pm 15.3$ & 5 & $4.0 \pm 6.0$ & 0.909 \\
\hline Decrease in eGFR \% & 95 & $4.2 \pm 14.9$ & 5 & $4.6 \pm 8.0$ & 0.951 \\
\hline $\begin{array}{l}\text { BMI - body mass ir } \\
\text { glomerular filtration } \\
\text { RPN for nonf } \\
\\
{ }^{\dagger} 12 /\end{array}$ & ex, $\mathrm{E}$ & $\begin{array}{l}\mathrm{BL} \text { - estimatec } \\
\mathrm{WIT} \text { - warm is } \\
\text { ning upper mo }\end{array}$ & & $\begin{array}{l}\text { oss, eGFR - estir } \\
\text { ime, *one patier } \\
\text { is not included. } \\
\text { imping }\end{array}$ & $\begin{array}{l}\text { mated } \\
\text { nt had }\end{array}$ \\
\hline
\end{tabular}

Table 4 - Comparison between first 50 cases and subsequent 51 cases.

\begin{tabular}{|c|c|c|c|c|c|}
\hline Variables & & $\begin{array}{c}\text { Cases } 1-50 \\
\mathrm{n} \quad(\%)\end{array}$ & & $\begin{array}{c}\text { ses } 51-101 \\
\mathrm{n} \quad(\%)\end{array}$ & $P$-value \\
\hline \multicolumn{6}{|l|}{ Gender } \\
\hline Female & \multicolumn{2}{|r|}{$20(19.8)$} & \multicolumn{2}{|r|}{$22(21.8)$} & 0.841 \\
\hline Male & \multicolumn{2}{|r|}{$30(29.7)$} & \multicolumn{2}{|r|}{$29(28.7)$} & \\
\hline \multicolumn{5}{|l|}{ Conversion to open } & \\
\hline No & \multicolumn{2}{|r|}{$46(45.5)$} & \multicolumn{2}{|r|}{$46(45.5)$} & 1.000 \\
\hline Yes & \multicolumn{2}{|r|}{$4(4.0)$} & \multicolumn{2}{|r|}{$5(5.0)$} & \\
\hline \multicolumn{5}{|l|}{ Transfusion } & \\
\hline No & \multicolumn{2}{|r|}{$50(49.5)$} & \multicolumn{2}{|r|}{$47(46.5)$} & 0.118 \\
\hline Yes & \multicolumn{2}{|r|}{$0 \quad(0.0)$} & \multicolumn{2}{|r|}{$4 \quad(4.0)$} & \\
\hline \multicolumn{5}{|l|}{ Off clamp technique } & \\
\hline No & \multicolumn{2}{|r|}{$44(43.6)$} & \multicolumn{2}{|r|}{$45(44.6)$} & 1.000 \\
\hline Yes & \multicolumn{2}{|r|}{$6 \quad(5.9)$} & \multicolumn{2}{|r|}{$6 \quad(5.9)$} & \\
\hline \multicolumn{5}{|l|}{ Surgical margins } & \\
\hline Negative & \multicolumn{2}{|r|}{$47(47.0)$} & \multicolumn{2}{|r|}{$48(48.0)$} & 1.000 \\
\hline Positive & \multicolumn{2}{|r|}{$3(3.0)$} & & $2(2.0)$ & \\
\hline Minor complication & & & & & \\
\hline No & & $42(41.6)$ & & $43(42.6)$ & 1.000 \\
\hline Yes & & $8 \quad(7.9)$ & & $8 \quad(7.9)$ & \\
\hline Major complication & & & & & \\
\hline No & & $48(47.5)$ & & $50(49.5)$ & 0.617 \\
\hline Yes & & $2(2.0)$ & & $1 \quad(1.0)$ & \\
\hline Any complication & & & & & \\
\hline No & & $41(40.6)$ & & $42(41.6)$ & 1.000 \\
\hline Yes & & $9 \quad(8.9)$ & & $9 \quad(8.9)$ & \\
\hline Trifecta achieved ${ }^{*}$ & & & & & \\
\hline No & & $37(37.0)$ & & $36(36.0)$ & 1.000 \\
\hline Yes & & $13(13.0)$ & & $14(14.0)$ & \\
\hline Histopathology & & & & & \\
\hline Benign & & $8 \quad(7.9)$ & & $13(12.9)$ & 0.327 \\
\hline Malignant & & $42(41.6)$ & & 38 (37.6) & \\
\hline & $\mathrm{n}$ & Mean \pm SD & $\mathbf{n}$ & Mean \pm SD & \\
\hline BMI & 50 & $30.1 \pm 5.9$ & 51 & $30.3 \pm 6.1$ & 0.820 \\
\hline $\begin{array}{l}\text { Age at diagnosis } \\
\text { (years) }\end{array}$ & 50 & $48.0 \pm 13.5$ & 51 & $49.3 \pm 14.0$ & 0.627 \\
\hline $\begin{array}{l}\text { Size of lesion }(\max \\
\text { diameter in } \mathrm{cm})\end{array}$ & 50 & $2.9 \pm 1.0$ & 51 & $3.2 \pm 1.1$ & 0.116 \\
\hline Renal score $\dagger$ & 50 & $5.9 \pm 1.7$ & 50 & $5.8 \pm 1.6$ & 0.723 \\
\hline $\begin{array}{l}\text { Procedure time } \\
\text { (minutes) }\end{array}$ & 50 & $151.2 \pm 48.7$ & 51 & $188.3 \pm 57.0$ & 0.001 \\
\hline $\mathrm{EBL}(\mathrm{ml})$ & 50 & $225.1 \pm 148.1$ & 51 & $287.3 \pm 260.0$ & 0.144 \\
\hline WIT (minutes) $\ddagger$ & 44 & $17.1 \pm 5.1$ & 45 & $18.1 \pm 7.0$ & 0.433 \\
\hline $\begin{array}{l}\text { Preoperative eGFR } \\
\mathrm{ml} / \mathrm{m}^{2}\end{array}$ & 50 & $94.7 \pm 24.2$ & 51 & $97.6 \pm 24.4$ & 0.544 \\
\hline $\begin{array}{l}\text { Postoperative eGFR } \\
\left(\mathrm{ml} / \mathrm{m}^{2}\right)\end{array}$ & 50 & $94.8 \pm 24.2$ & 51 & $87.2 \pm 22.7$ & 0.104 \\
\hline $\begin{array}{l}\text { Decrease in eGFR } \\
\left(\mathrm{ml} / \mathrm{m}^{2}\right)\end{array}$ & 50 & $-0.2 \pm 14.7$ & 51 & $10.4 \pm 14.3$ & 0.000 \\
\hline $\begin{array}{l}\text { Decrease in eGFR } \\
\%\end{array}$ & 50 & $-1.3 \pm 14.9$ & 51 & $10.1 \pm 12.5$ & 0.000 \\
\hline $\begin{array}{l}\text { BMI - body mass in } \\
\text { glomerular filtration } \\
\text { of any complication, } \\
\text { †One patient had } \mathrm{F} \\
\text { included. } \neq \mathrm{n}\end{array}$ & $\begin{array}{l}\mathrm{E} \\
\mathrm{W} \\
\text { tiv } \\
\text { Jf }\end{array}$ & $\begin{array}{l}\text { EBL - estimated } \\
\text { WIT - warm isch } \\
\text { ive surgical marg } \\
\text { for nonfunction } \\
12 \text { patients had }\end{array}$ & & $\begin{array}{l}\text { ss, eGFR - es } \\
\text { ne, *Trifecta - } \\
\text { schemia time } \\
\text { er moiety and } \\
\text { ular clamping }\end{array}$ & $\begin{array}{l}\text { mated } \\
\text { reedom } \\
25 \text { min. } \\
\text { s not }\end{array}$ \\
\hline
\end{tabular}


Table 5 - Tumor characteristics: Comparison of current series with others reporting $\geq 100$ patients.

\begin{tabular}{|c|c|c|c|c|c|c|c|c|}
\hline \multirow[t]{2}{*}{ Study } & \multirow[t]{2}{*}{$\begin{array}{c}\text { Centers } \\
(\mathbf{n})\end{array}$} & \multirow[t]{2}{*}{ Years } & \multirow[t]{2}{*}{$\begin{array}{l}\text { Patient } \\
\text { number }\end{array}$} & \multirow[t]{2}{*}{$\begin{array}{c}\text { Tumor } \\
\text { category }\end{array}$} & \multicolumn{2}{|c|}{$\begin{array}{l}\text { Tumor size } \\
(\mathrm{cm})\end{array}$} & \multicolumn{2}{|c|}{ Renal score } \\
\hline & & & & & Mean \pm SD & Median (range) & Mean \pm SD & Median (range) \\
\hline Benway et $\mathrm{al}^{6}$ & Multicenter (3) & $2004-2008$ & 129 & & 2.9 & & & \\
\hline Scoll et $\mathrm{al}^{7}$ & Single institution & $2007-2009$ & 100 & & & $2.8(1-8)$ & $6.8 \pm 1.7^{*}$ & $7(4-10)^{*}$ \\
\hline Kaouk et $\mathrm{al}^{8}$ & Single institution & $2006-2011$ & 400 & & $3.17 \pm 1.64$ & & $7.2 \pm 2$ & \\
\hline Tanagho et $\mathrm{al}^{9}$ & Multicenter (5) & $2007-2011$ & 886 & & $3 \pm 1.6$ & & $6.9 \pm 2$ & \\
\hline Ficarra et $\mathrm{al}^{10}$ & Multicenter (4) & $2008-2010$ & 200 & & & $2.8(1.9-3.5)$ & & \\
\hline Minervini et $\mathrm{al}^{11}$ & Multicenter (6) & $2010-2011$ & 105 & & $2.8 \pm 1.5$ & & & $7(6-7)^{*}$ \\
\hline Oh et $\mathrm{al}^{12}$ & Single surgeon & $2003-2013$ & 100 & & $2.52 \pm 1.26$ & $(0.90-6.00)$ & & $7(6-9)$ \\
\hline Kim et $\mathrm{al}^{13}$ & Multicenter (5) & $2003-2011$ & 195 & & $2.35 \pm 1.16$ & & & \\
\hline Lista et $\mathrm{al}^{14}$ & Multicenter (3) & $2006-2012$ & 339 & & & $2.7(1-7)$ & & $8(6-13)^{\dagger}$ \\
\hline Maddox et $\mathrm{al}^{15}$ & Single institution & $2008-2013$ & 241 & T1b-T3 & & $5(4.1-5.2)$ & $7.1 \pm 2.2$ & \\
\hline Zargar et $\mathrm{al}^{1}$ & Multiple (5) & $2004-2013$ & 1185 & & & $2.3(1.3)$ & & $7(3)$ \\
\hline \multirow{4}{*}{ Abdel Raheem et al ${ }^{16}$} & Single surgeon & $2006-2015$ & 295 & & & & & \\
\hline & & & 72 & PADUA $\leq 7$ & & $2(1.5-2.7)$ & & \\
\hline & & & 102 & PADUA 8-9 & & $2.9(1.8-3.9)$ & & \\
\hline & & & 121 & PADUA $\geq 10$ & & $4.1(2.9-5.3)$ & & \\
\hline \multirow[t]{3}{*}{ Janda et al ${ }^{17}$} & Single institution & $2008-2014$ & 232 & & & & & \\
\hline & & & 168 & T1a & & $2.6(2.0-3.1)^{\ddagger}$ & $6.9 \pm 2.01$ & \\
\hline & & & 64 & $\mathrm{~T} 1 \mathrm{~b}$ & & $4.8(4.5-5.6)^{\ddagger}$ & $8.2 \pm 1.62$ & \\
\hline Peyronnet et a ${ }^{18}$ & Multicenter (6) & $2006-2014$ & 937 & & $3.3 \pm 0.6$ & & $6.8 \pm 0.1$ & \\
\hline Potretzke et $\mathrm{al}^{19}$ & Single institution & $2007-2014$ & 286 & & $2.8 \pm 1.4$ & & $7.4 \pm 1.9$ & \\
\hline Xie et $\mathrm{a}^{20}$ & Single surgeon & $2013-2014$ & 144 & & $3.1 \pm 2.0$ & & $6.7 \pm 2.0$ & \\
\hline Han et $\mathrm{al}^{21}$ & Single institution & $2011-2014$ & 147 & & $2.58 \pm 1.13$ & & $6.58 \pm 1.80$ & \\
\hline Luciani et al ${ }^{22}$ & Single surgeon & $2012-2016$ & 110 & & & $3.5(2.5-4.8)$ & & \\
\hline \multirow[t]{2}{*}{ Maurice et $\mathrm{al}^{23}$} & Single center & $2011-2015$ & 301 & T1a & & $2.7(2.0-3.3)$ & & $7(5-8)$ \\
\hline & & & 114 & $\mathrm{~T} 1 \mathrm{~b}$ & & $5.0(4.4-5.7)$ & & $9(7-10)$ \\
\hline Moskowitz et $\mathrm{al}^{24}$ & Multicenter (4) & $2008-2015$ & 1139 & & & $3.0(2.1-4.0)$ & & $7.0(6.0-9.0)$ \\
\hline Paulucci et $\mathrm{al}^{25}$ & Multicenter (4) & $2008-2016$ & $960^{\S}$ & & & $3(2.1-4)$ & & $7(6-9)$ \\
\hline Veeratterapillay et $\mathrm{a}^{26}$ & Multicenter (4) & $2012-2015$ & 250 & & $3.1 \pm 1$ & & $6.1 \pm 2$ & \\
\hline Current study & Single center & $2008-2018$ & 101 & $\mathrm{~T} 1 \mathrm{a}-\mathrm{T} 1 \mathrm{~b}$ & $3.1 \pm 1$ & $3(1.3-6.4)$ & $5.8 \pm 1.7$ & $6(4-10)$ \\
\hline
\end{tabular}

(3\%) patients had a complication grade $\geq$ III, ClavienDindo classification. One had an arteriovenous fistula requiring embolization 3 weeks postoperatively, one had a diaphragmatic injury requiring intraoperative repair, and one patient developed atrial fibrillation requiring intensive care admission. A total of $73(73 \%)$ patients achieved a trifecta of freedom from any complication, negative surgical margins, and ischemia time $\leq 25 \mathrm{~min}$. The outcomes of WIT, EBL, conversion to open and trifecta achievement were not different between the first and subsequent 50 patients (Table 4). Significantly longer operative time and more decrease in eGFR were found in the latter group.

Discussion. This initial experience of RPN in one Middle Eastern country demonstrates outcomes comparable to Western series reporting on at least
100 RPN (Tables 5-7). ${ }^{1,6-26}$ Our initial 101 cases had a slightly lower median renal score of 6 and a mean score of 5.8 compared to 22 studies reporting a median renal score between 7 and 9 and a mean score ranging between 6 and 8.2. This tendency to select less complex renal masses for RPN reflects the initial experience of our surgeons embarking on the procedure. Tumor size, however, in the current series was comparable to other studies. The median tumor size in the current series was $3 \mathrm{~cm}$ and the mean was $3.1 \mathrm{~cm}$ compared to a range of median size of 2 to $5 \mathrm{~cm}$ and mean of 2.4 to $3.3 \mathrm{~cm}$ reported in other studies. The smallest tumor in the current series was $1.3 \mathrm{~cm}$ in diameter, whereas in other studies the smallest reported tumor was $0.9 \mathrm{~cm}$. Remarkably, operative and postoperative parameters gauging the surgeons' skills in performing $\mathrm{RPN}$ was on par with those reporting larger series 
Table 6 - Comparison of current series with others reporting $\geq 100$ patients.

\begin{tabular}{|c|c|c|c|c|c|c|c|}
\hline \multirow[t]{2}{*}{ Study } & \multicolumn{2}{|c|}{ OR time (min) } & \multicolumn{2}{|c|}{ WIT (min) } & \multirow{2}{*}{$\begin{array}{c}\text { Unclamped } \\
\mathrm{n}(\%)\end{array}$} & \multicolumn{2}{|c|}{$\mathrm{EBL}(\mathrm{mL})$} \\
\hline & Mean \pm SD & Median (range) & Mean \pm SD) & Median (range) & & Mean (SD) & Median (range) \\
\hline Benway et $\mathrm{al}^{6}$ & 189 & & 19.7 & & & 155 & \\
\hline Scoll et $\mathrm{al}^{7}$ & 206 & $203(85-369)$ & 25.5 & $25.5(0-53)$ & $12(12.0)$ & 127 & $50(50-800)$ \\
\hline Kaouk et $\mathrm{al}^{8}$ & $190.3 \pm 57$ & & $19.2 \pm 10.7$ & & $36 \quad(9.0)$ & 260 & \\
\hline Tanagho et $\mathrm{al}^{9}$ & $183.6 \pm 60.4$ & & $18.8 \pm 9$ & & $66 \quad(7.5)$ & $181(208.9)$ & $100(100-250)^{*}$ \\
\hline Ficarra et $\mathrm{al}^{10}$ & & $120(90-157)$ & & $18(14-23)$ & $20(10.0)$ & & $100(50-150)$ \\
\hline Minervini et $\mathrm{al}^{11}$ & $168 \pm 56$ & & $18.2 \pm 7$ & & $40(38.0)$ & $125(128)$ & \\
\hline Oh et $\mathrm{al}^{12}$ & $182.89 \pm 83.98$ & & $21.86 \pm 9.3$ & & & $212.04(160.8)$ & \\
\hline Kim et $\mathrm{al}^{13}$ & & $135(110-170)$ & $23.82 \pm 12.0$ & & Excluded & & $200(100-300)$ \\
\hline Lista et $\mathrm{al}^{14}$ & $141.7 \pm 130$ & $130(60-350)$ & 17.8 & $17(7-51)$ & 0 & 136.6 & $100(30-1600)$ \\
\hline Maddox et $\mathrm{al}^{15}$ & & $183(156-220)$ & & $20.5(17-25)$ & & & $200(100-300)$ \\
\hline Zargar et $\mathrm{al}^{1}$ & $168 \pm 68$ & & & $18(9)$ & & & $100(125)$ \\
\hline \multicolumn{8}{|l|}{ Abdel Raheem et al ${ }^{16}$} \\
\hline & & $149(107-180)$ & & $23(18-27)$ & $26(36.1)$ & & $200(100-332)$ \\
\hline & & $163(128-196)$ & & $24(18-30)$ & $20(19.6)$ & & $275(130-563)$ \\
\hline & & $164(119-219)$ & & $26(22-32)$ & $5(4.2)$ & & $360(200-550)$ \\
\hline \multicolumn{8}{|l|}{ Janda et $\mathrm{al}^{17}$} \\
\hline & & $179(153-210)$ & & $21(16-26)$ & $9(5.0)$ & & $100(75-250)$ \\
\hline & & $210(182-237)$ & & $23(19-31)$ & $0 \quad(0)$ & & $200(100-325)$ \\
\hline Peyronnet et $\mathrm{al}^{18}$ & $153.2 \pm 2$ & & $15.7 \pm 0.3$ & & $61 \quad(6.6)$ & $275.1(13)$ & \\
\hline Potretzke et al ${ }^{19}$ & $154.8 \pm 49.4$ & & $19.8 \pm 8.7$ & & $(25.3)$ & $179.7(229.1)$ & \\
\hline Xie et $\mathrm{al}^{20}$ & $118.7 \pm 34.4$ & & $17.9 \pm 6.4$ & & & $86.5(87.5)$ & \\
\hline Han et $\mathrm{al}^{21}$ & $162.3 \pm 32.2$ & & $24.7 \pm 7.3$ & & & & \\
\hline Luciani et $\mathrm{al}^{22}$ & & $200(120-385)$ & & $22(0-45)$ & & & $150(0-900)$ \\
\hline \multirow[t]{2}{*}{ Maurice et $\mathrm{al}^{23}$} & & & & $17(13-23)$ & & & \\
\hline & & & & $26(20-30)$ & & & \\
\hline Moskowitz et $\mathrm{al}^{24}$ & & $183.0(151.0-224.0)$ & & $15.0(11.0-20.0)$ & & & $100.0(50.0-150.0)$ \\
\hline Paulucci et $\mathrm{al}^{25}$ & & $179(150-220)$ & & $16(12.5-21)$ & $94(10.4)$ & & $100(50-150)$ \\
\hline Veeratterapillay et $\mathrm{a}^{26}$ & $141 \pm 38^{\dagger}$ & & $16.7 \pm 8$ & & & $205(145)$ & \\
\hline Current study & $170.4 \pm 57.4$ & $166(66-381)$ & $17.6 \pm 6.1$ & $17(8-40)$ & $12(11.9)$ & $256.5(213.4)$ & $200(5-1500)$ \\
\hline
\end{tabular}

beyond the learning curve. The median WIT in the current series was 17 minutes and the mean was 17.6 in comparison to other studies with a median range of WIT of 15-26 minutes and a mean range of 15.7-25.5 minutes. The off-clamp RPN constituted $11.9 \%$ of the current series compared to a range of $0-38 \%$ reported by others. The operative time, EBL, and LOS were comparable to other reported series. The current series had $5 \%$ positive surgical margin (PSM) compared to a range of $0-9.9 \%$ reported by others. Except for older age, we found no significant risk factor associated with PSM. Any complication was reported only in 3\% of cases. This favorable outcome is among the lowest reported by other series, ranging between $0.4 \%$ and $39 \%$. The development of a complication or the longer procedure time were risk factors for a decrease of eGFR $\leq 15 \%$ even in face of a higher preoperative eGFR. We did not factor in the analysis risk factors for decreased eGFR such as diabetes, hypertension, dyslipidemia or nephrotoxic medications. As these risk factors likely contributed to the preoperative eGFR, we think that for the purpose of a short-term perioperative analysis of eGFR change as a surrogate for quality of surgery, our conclusions are accurate. To evaluate the impact of RPN on renal function in the long term, these risk factors among other confounders of the renal functional reserve are worthwhile to study. The current series reported the highest trifecta, $73 \%$, in comparison to the 5 studies reporting a trifecta outcome ranging between $37.5 \%$ and $72.2 \%$. On a different note, the conversion rate to open surgery was the second most common in all studies reviewed. Conversion to open partial nephrectomy in the current series was $8.9 \%$, and no case was converted to nephrectomy. In comparison, other series reported conversion to open nephrectomy, either to partial or total, in $0-11.7 \%$.

Although the number of patients who underwent RPN was relatively small per surgeon and spanning a long period compared to higher-volume centers, the results indicate that the number of surgeries needed to gain the cumulative learning experience is small. Comparison of the first 50 cases with the subsequent 
Robotic partial nephrectomy in KSA ... Seyam et al

Table 7 - Postoperative outcome: Comparison of current series with others reporting $\geq 100$ patients.

\begin{tabular}{|c|c|c|c|c|c|c|}
\hline \multirow[t]{2}{*}{ Study } & \multicolumn{2}{|c|}{ LOS (days) } & \multirow{2}{*}{$\begin{array}{l}\text { PSM } \\
\text { n (\%) }\end{array}$} & \multirow{2}{*}{$\begin{array}{c}\text { Conv. OPN } \\
\mathrm{n}(\%)\end{array}$} & \multirow{2}{*}{$\begin{array}{l}\text { Comp. } \\
\text { n (\%) }\end{array}$} & \multirow{2}{*}{$\begin{array}{c}\text { Trifecta* } \\
\text { n (\%) }\end{array}$} \\
\hline & Mean $(\mathrm{SD})$ & Median (range) & & & & \\
\hline Benway et $\mathrm{al}^{6}$ & 2.4 & & $5(3.9)$ & $2(3.3)$ & $11(18.3)$ & \\
\hline Scoll et $\mathrm{al}^{7}$ & 3.2 & $3(1-7)$ & $5(5.7)$ & $2(2.0)$ & $11(11.0)$ & \\
\hline Kaouk et $\mathrm{al}^{8}$ & 3.6 & & $9(2.3)$ & $6(1.5)$ & $61(15.3)$ & \\
\hline Tanagho et $\mathrm{al}^{9}$ & & & & 7 (11.7) & $139(13.0)$ & \\
\hline Ficarra et $\mathrm{al}^{10}$ & $6(5-6)$ & & $9 / 158(5.7)$ & & $28 / 200(14.0)$ & \\
\hline Minervini et $\mathrm{a}^{11}$ & & $5(4-6)$ & $6(5.7)$ & $1(1.0)$ & $1(1.0) \dagger$ & \\
\hline Oh et $\mathrm{al}^{12}$ & $5.4(1.8)$ & & $0(0.0)$ & & $10(10.0)$ & \\
\hline Kim et $\mathrm{al}^{13}$ & & & $3(1.5)$ & Excluded & & \\
\hline Lista et $\mathrm{al}^{14}$ & & & $22(6.5)$ & $3(0.9)$ & $49(14.5)$ & \\
\hline Maddox et $\mathrm{al}^{15}$ & & & $3(6.8)$ & & $1 \quad(0.4)$ & \\
\hline 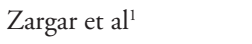 & & & $38(3.2)$ & $2(0.2)^{\ddagger}$ & $192(16.2)$ & $829(70.0)$ \\
\hline \multicolumn{7}{|l|}{ Abdel Raheem et al ${ }^{16}$} \\
\hline & & $5(4-5)$ & $3(4.1)$ & $0^{\ddagger}$ & $7 \quad(9.7)$ & $47(65.3)$ \\
\hline & & $5(5-7)$ & $6(5.8)$ & $1(1.0)^{\ddagger}$ & $26(25.5)$ & $58(56.9)$ \\
\hline & & $5(5-8)$ & $12(9.9)$ & $7(5.9)^{\ddagger}$ & $26(21.5)$ & $45(37.5)$ \\
\hline \multicolumn{7}{|l|}{ Janda et $\mathrm{al}^{17}$} \\
\hline & & $1.7(0.86)$ & $10(6.0)$ & $5(3.0)$ & $61(36.0)$ & \\
\hline & & $2.2(2.51)$ & $4(6.0)$ & $0(0)$ & $25(39.0)$ & \\
\hline Peyronnet et a ${ }^{18}$ & $4.7(0.2)$ & & $48(5.2)$ & & $168(17.9)$ & \\
\hline Potretzke et $\mathrm{al}^{19}$ & $2.2(1.1)$ & & $(6.0)$ & & (9.9) & \\
\hline Xie et $\mathrm{al}^{20}$ & $6.3(2.1)$ & & $2(1.4)$ & & $14 \quad(9.7)$ & $90(62.5)^{\S}$ \\
\hline Han et $\mathrm{al}^{21}$ & $5.3(1.4)$ & & & & $5(3.4)$ & \\
\hline Luciani et $\mathrm{a}^{22}$ & & $6(4-22)$ & $7(6.3)$ & & $8(7.3)^{\dagger}$ & \\
\hline \multirow[t]{2}{*}{ Maurice et $\mathrm{a}^{23}$} & & & $12(4.1)$ & & $19(6.3)^{\dagger}$ & $199(66.1)$ \\
\hline & & & $7(6.2)$ & & $4(3.5)^{\dagger}$ & $46(40.4)$ \\
\hline Moskowitz et $\mathrm{a}^{24}$ & & $1.0(1.0-2.0)$ & $50(5.2)$ & & $130(11.3)$ & \\
\hline Paulucci et $\mathrm{al}^{25}$ & & $1(1-2)$ & $30(4)$ & & $115(12.0)$ & $484(72.2)$ \\
\hline Veeratterapillay et a ${ }^{26}$ & & & $(7.3)$ & $5(2.0)$ & $(16.4)$ & \\
\hline Current study & $3.8(1.4)$ & $4(2-14)$ & $5(5)^{* *}$ & $9(8.9)$ & $3(3.0)^{\dagger}$ & $73(73.0)$ \\
\hline $\begin{array}{r}\text { Comp. - complicatio } \\
\text { from any complica } \\
\text { complication: grade } \\
\text { negative, (2) warm isc } \\
\text { classificatio }\end{array}$ & $\begin{array}{l}\text { Oonv. - convers } \\
\text { (ii) negative st } \\
\text { Clavien-Dindo } \\
\text { a time (WIT) } \\
\text { *One patient v }\end{array}$ & $\begin{array}{l}\text { s, LOS - length of } \\
\text { ical margins, and ( } \\
\text { mplications, ‡Con } \\
20 \text { min, and (3) } r \\
\text { RPN for nonfun }\end{array}$ & $\begin{array}{l}\text { SM - positive } \\
\text { hemia time } \leq \\
\text { n to radical n } \\
\text { jor complicati } \\
\text { hg upper rena }\end{array}$ & $\begin{array}{l}\text { gical margin, } \\
\text { in (including } \\
\text { ectomy, \$MI } \\
\text { occur (grades } \\
\text { iety was exclu }\end{array}$ & $\begin{array}{l}\text { fecta was definec } \\
\mathrm{d} \text { or warm ischer } \\
\text { ore (1) the surgic } \\
\text { according to the } \\
\text { from the calcula }\end{array}$ & $\begin{array}{l}\text { (i) freedom } \\
\text { † } † \text { Major } \\
\text { argins are } \\
\text { ivien-Dindo } \\
\end{array}$ \\
\hline
\end{tabular}

surgeries showed no significant difference in the WIT, EBL, complication rate, conversion to open surgery, PSM or achievement of a trifecta benchmark all of which indicate no appreciable change in the quality of surgery over the protracted period. Admittedly the duration of surgery increased, and this may have contributed more significant decrease in post-operative eGFR. The longer duration of surgery might be due to a more difficult surgery beyond the actual time and skill spent to excise the tumor from the kidney. Different factors contributed to the favorable outcome of the current series. The first factor was that all surgeons had prior experience with laparoscopic partial nephrectomy and robotic nephrectomy. These findings on the transition from laparoscopic to robotic partial nephrectomy are similar to other reports from single surgeon series at high-volume centers. ${ }^{27}$ Second, 2 experienced surgeons teamed up in a single case. We believe it is important for outcomes of RPN to be reported from various parts of the world, thus attesting to the generalizability of the robotic technique as well as bringing the benefits of robotic technology to Middle Eastern patients. Study limitations include the retrospective design and small cohort size. Future studies from our region may include long-term functional and oncological outcomes of RPN.

In conclusions, our initial experience of robotic partial nephrectomy is associated with a surgical outcome comparable to that reported by higher volume centers. The favorable outcome reflects that the number of surgeries needed to gain the cumulative learning experience is small, even with a protracted course of time. 
Acknowledgment. We would like to thank Elsevier Publishing Company, Amsterdam, Netherlands (webshop.elsevier.com/ languageservices/languageediting) for English language editing.

\section{References}

1. Zargar H, Allaf ME, Bhayani S, Stifelman M, Rogers C, Ball MW, et al. Trifecta and optimal perioperative outcomes of robotic and laparoscopic partial nephrectomy in surgical treatment of small renal masses: a multi-institutional study. BJU Int 2015; 116: 407-414.

2. Choi JE, You JH, Kim DK, Rha KH, Lee SH. Comparison of perioperative outcomes between robotic and laparoscopic partial nephrectomy: a systematic review and meta-analysis. Eur Urol 2015; 67: 891-901.

3. Ghani KR, Sukumar S, Sammon JD, Rogers CG, Trinh QD, Menon M. Practice patterns and outcomes of open and minimally invasive partial nephrectomy since the introduction of robotic partial nephrectomy: results from the nationwide inpatient sample. J Urol 2014; 191: 907-912.

4. Ellison JS, Montgomery JS, Wolf JSJ, Hafez KS, Miller DC, Weizer AZ. A matched comparison of perioperative outcomes of a single laparoscopic surgeon versus a multisurgeon robotassisted cohort for partial nephrectomy. J Urol 2012; 188: 45-50.

5. Levey AS, Bosch JP, Lewis JB, Greene T, Rogers N, Roth D. A more accurate method to estimate glomerular filtration rate from serum creatinine: a new prediction equation. Modification of Diet in Renal Disease Study Group. Ann Intern Med 1999; 130: 461-470.

6. Benway BM, Bhayani SB, Rogers CG, Dulabon LM, Patel MN, Lipkin M, et al. Robot assisted partial nephrectomy versus laparoscopic partial nephrectomy for renal tumors: a multiinstitutional analysis of perioperative outcomes. J Urol 2009; 182: 866-872.

7. Scoll BJ, Uzzo RG, Chen DYT, Boorjian SA, Kutikov A, Manley BJ, et al. Robot-assisted partial nephrectomy: a large single-institutional experience. Urology 2010; 75: 1328-1334.

8. Kaouk JH, Khalifeh A, Hillyer S, Haber GP, Stein RJ, Autorino R. Robot-assisted laparoscopic partial nephrectomy: step-bystep contemporary technique and surgical outcomes at a single high-volume institution. Eur Urol 2012; 62: 553-561.

9. Tanagho YS, Kaouk JH, Allaf ME, Rogers CG, Stifelman MD, Kaczmarek BF, et al. Perioperative complications of robot-assisted partial nephrectomy: analysis of 886 patients at 5 United States centers. Urology 2013; 81: 573-579.

10. Ficarra V, Minervini A, Antonelli A, Bhayani S, Guazzoni G, Longo N, et al. A multicentre matched-pair analysis comparing robot-assisted versus open partial nephrectomy. BJU Int 2014; 113: 936-941.

11. Minervini A, Vittori G, Antonelli A, Celia A, Crivellaro S, Dente $\mathrm{D}$, et al. Open versus robotic-assisted partial nephrectomy: a multicenter comparison study of perioperative results and complications. World J Urol 2014; 32: 287-293.

12. Oh JJ, Byun S, Hong SK, Jeong CW, Lee SE. Comparison of robotic and open partial nephrectomy: Single-surgeon matched cohort study. Can Urol Assoc J 2014; 8: E471-E475.

13. Kim JH, Park YH, Kim YJ, Kang SH, Byun SS, Kwak C, et al. Perioperative and long-term renal functional outcomes of robotic versus laparoscopic partial nephrectomy: a multicenter matched-pair comparison. World J Urol 2015; 33: 1579-1584.
14. Lista G, Buffi NM, Lughezzani G, Lazzeri M, Abrate A, Mistretta A, et al. Margin, ischemia, and complications system to report perioperative outcomes of robotic partial nephrectomy: a European Multicenter Observational Study (EMOS project). Urology 2015; 85: 589-595.

15. Maddox M, Mandava S, Liu J, Boonjindasup A, Lee BR. Robotic partial nephrectomy for clinical stage T1b tumors: intermediate oncologic and functional outcomes. Clin Genitourin Cancer 2015; 13: 94-99.

16. Abdel Raheem A, Alatawi A, Kim DK, Sheikh A, Alabdulaali I, Han WK, et al. Outcomes of high-complexity renal tumours with a Preoperative Aspects and Dimensions Used for an Anatomical (PADUA) score of $\geq 10$ after robot-assisted partial nephrectomy with a median 46.5-month follow-up: a tertiary centre experience. BJU Int 2016; 118: 770-778.

17. Janda G, Deal A, Yang H, Nielsen M, Smith A, Pruthi RS, et al. Single-Institution Experience with Robotic Partial Nephrectomy for Renal Masses Greater Than $4 \mathrm{~cm}$. J Endourol 2016; 30: 384-389.

18. Peyronnet B, Seisen T, Oger E, Vaessen C, Grassano Y, Benoit T, et al. Comparison of 1800 Robotic and Open Partial Nephrectomies for Renal Tumors. Ann Surg Oncol 2016; 23 : 4277-4283.

19. Potretzke AM, Potretzke TA, Knight BA, Vetter J, Park AM, Anderson G, et al. Tumor diameter accurately predicts perioperative outcomes in T1 renal cancer treated with robot-assisted partial nephrectomy. World J Urol 2016; 34: 1643-1650.

20. Xie Y, Ma X, Gu L, Li H, Lv X, Gao Y, et al. Associating the learning curve and tumor anatomical complexity with the margins, ischemia, and complications rate after robot-assisted partial nephrectomy. Int J Surg Lond Engl 2016; 36: 219-224.

21. Han KS, Song GH, You D, Song C, Jeong IG, Hong JH, et al. Comparison of hand-assisted laparoscopic vs robot-assisted laparoscopic vs open partial nephrectomy in patients with T1 renal masses. J Endourol 2017; 31: 374-379.

22. Luciani LG, Chiodini S, Mattevi D, Cai T, Puglisi M, Mantovani W, et al. Robotic-assisted partial nephrectomy provides better operative outcomes as compared to the laparoscopic and open approaches: results from a prospective cohort study. J Robot Surg 2017; 11: 333-339.

23. Maurice MJ, Ramirez D, Kara O, Malkoc E, Nelson RJ, Fareed K, et al. Optimum outcome achievement in partial nephrectomy for T1 renal masses: a contemporary analysis of open and robot-assisted cases. BJU Int 2017; 120: 537-543.

24. Moskowitz EJ, Paulucci DJ, Reddy BN, Blum KA, Rosen DC, Abaza R, et al. Predictors of medical and surgical complications after robot-assisted partial nephrectomy: An analysis of 1139 patients in a multi-institutional kidney cancer database. $J$ Endourol 2017; 31: 223-238.

25. Paulucci DJ, Abaza R, Eun DD, Hemal AK, Badani KK. Robot-assisted partial nephrectomy: continued refinement of outcomes beyond the initial learning curve. BJU Int 2017; 119 : 748-754.

26. Veeratterapillay R, Addla SK, Jelley C, Bailie J, Rix D, Bromage $S$, et al. Early surgical outcomes and oncological results of robot-assisted partial nephrectomy: a multicentre study. BJU Int 2017; 120: 550-555.

27. Dube H, Bahler CD, Sundaram CP. The learning curve and factors affecting warm ischemia time during robot-assisted partial nephrectomy. Indian J Urol 2015; 31: 223-228. 